DESY 08-127

September 2008

\title{
Superparticle Mass Window from Leptogenesis and Decaying Gravitino Dark Matter
}

\author{
Wilfried Buchmüller, Motoi Endo and Tetsuo Shindou \\ Deutsches Elektronen-Synchrotron DESY, Hamburg, Germany
}

\begin{abstract}
Gravitino dark matter, together with thermal leptogenesis, implies an upper bound on the masses of superparticles. In the case of broken R-parity the constraints from primordial nucleosynthesis are naturally satisfied and decaying gravitinos lead to characteristic signatures in high energy cosmic rays. We analyse the implications for supergravity models with universal boundary conditions at the grand unification scale. Together with low-energy observables one obtains a window of superparticle masses, which will soon be probed at the LHC, and a range of allowed reheating temperatures.
\end{abstract}




\section{Introduction}

Standard thermal leptogenesis [1] provides a simple and elegant explanation of the origin of matter. It is a natural consequence of the seesaw mechanism, and it is perfectly consistent with the small neutrino masses inferred from neutrino oscillation data [2].

Thermal leptogenesis works without and with supersymmetry. In the latter case, however, there is a clash with the 'gravitino problem' [3-5]: the large temperature required by leptogenesis exceeds the upper bound on the reheating temperature from primordial nucleosynthesis $(\mathrm{BBN})$ in typical supergravity models with a neutralino as lightest superparticle (LSP) and an unstable gravitino. If the gravitino is the LSP, the condition that relic gravitinos do not overclose the universe yields an upper bound on the reheating temperature [6]. Furthermore, the next-to-lightest superparticle (NLSP) is long lived, and one has to worry about the effect of NLSP decays on nucleosynthesis.

It is remarkable that, despite these potential problems, a large leptogenesis temperature of order $10^{10} \mathrm{GeV}$ can account for the observed cold dark matter in terms of thermally produced relic gravitinos [7]. Requiring consistency with nucleosynthesis yields constraints on the superparticle mass spectrum. Due to improved analyses of BBN, the original proposal of a higgsino NLSP is no longer viable, and also other possible NLSPs are strongly constrained. The case of a stau NLSP is cornered by bounds following from catalyzed production of ${ }^{6} \mathrm{Li}$ [8], with the possible exception of a large left-right mixing in the stau sector [9]. In some models a sneutrino [10] or a stop [11] can still be a viable NLSP.

Recently, it has been shown that in the case of small R-parity and lepton number breaking, such that the baryon asymmetry is not erased by sphaleron processes [12], thermal leptogenesis, gravitino dark matter and primordial nucleosynthesis are naturally consistent [13]. Although the gravitino is no longer stable, its decay into standard model (SM) particles is doubly suppressed by the Planck mass and the small R-parity breaking parameter. Hence, its lifetime exceeds the age of the universe by many orders of magnitude, and it remains a viable dark matter candidate [14]. Gravitino decays lead to characteristic signatures in high energy cosmic rays. The produced flux of gamma-rays [13-17] and positrons [17,18] may explain the observed excess in the EGRET [19] and HEAT [20] data. This hypothesis will soon be tested by the satellite experiments FGST and PAMELA.

In this paper we study the implications of leptogenesis and gravitino dark matter with broken R-parity on the mass spectrum of superparticles. Since the unification of gauge couplings in the minimal supersymmetric extension of the standard model (MSSM) is one of the main motivations for low-energy supersymmetry, we shall focus on versions of the MSSM with universal boundary conditions for scalar and gaugino masses at the grand unification (GUT) scale. As we shall see, the corresponding spectrum of superparticle masses will be fully covered at the LHC. This is the main result of our analysis.

After some comments on R-parity violation in Section 2, we discuss the lower bound on the reheating temperature from leptogenesis and the upper bound on the NLSP mass from gravitino dark matter in Section 3. Section 4 deals with constraints on MSSM parameters from low-energy observables, and the results of our numerical analysis are presented in Section 5, followed by some conlusions in Section 6 . 


\section{Constraints on R-parity violation}

Phenomenological aspects of R-parity violation have been widely discussed in the literature [21]. Here we are interested in the case of small R-parity and lepton number breaking which was investigated in $[13,14,17]$. The details strongly depend on the flavour structure of Rparity violating couplings and the pattern of supersymmetry breaking. For completeness, we recall in the following the order of magnitude of bounds on R-parity violating couplings, the corresponding lifetimes of gravitino and NLSP, and in particular the dependence on the gravitino mass.

Stringent constraints on the lepton number and R-parity violating interactions

$$
W_{\Delta L=1}=\lambda_{i k j} l_{i} e_{j}^{c} l_{k}+\lambda_{k j i}^{\prime} d_{i}^{c} q_{j} l_{k}
$$

are imposed by baryogenesis. Both operators contain lepton doublets. Together with sphaleron processes they therefore influence the baryon asymmetry at high temperature in the early universe. The requirement that an existing baryon asymmetry is not erased before the electroweak transition typically implies [12]

$$
\lambda, \lambda^{\prime}<10^{-7} \text {. }
$$

Remarkably, for such a small breaking of R-parity a gravitino LSP has a lifetime much longer than the age of the universe [14] because of the double suppression of the decay rate by the inverse Planck mass and the R-parity breaking coupling. One then obtains for the gravitino lifetime (cf. [13])

$$
\tau_{3 / 2} \sim 10^{25} \mathrm{~s}\left(\frac{\lambda}{10^{-8}}\right)^{-2} \eta\left(\frac{\tilde{m}}{m_{3 / 2}}\right)\left(\frac{m_{3 / 2}}{100 \mathrm{GeV}}\right)^{-3},
$$

where $\widetilde{m} \sim \mathcal{O}(100 \mathrm{GeV})$ is a characteristic supersymmetry breaking mass scale. In the case of light gravitinos, $m_{3 / 2} \ll \widetilde{m}$, where only the decay into photon neutrino pairs is kinematically allowed, $\eta=1$ has been assumed in [13]. For heavier gravitinos, decays into W-boson lepton and Z-boson lepton pairs are also possible, and we only know that $\eta=\mathcal{O}(1)$ [17]. In particular, the relation between gravitino lifetime and gravitino mass depends on the pattern of supersymmetry breaking.

In the case of a small breaking of R-parity, with an unstable gravitino LSP, the NLSP lifetime becomes very short,

$$
c \tau_{\mathrm{NLSP}} \sim 10 \mathrm{~cm}\left(\frac{\lambda}{10^{-8}}\right)^{-2}\left(\frac{m_{\mathrm{NLSP}}}{100 \mathrm{GeV}}\right)^{-1} .
$$

For couplings $\lambda, \lambda^{\prime}>10^{-14}$, the NLSP lifetime becomes shorter than $10^{3} \mathrm{~s}$. In case of a stau NLSP, superparticle decays then do not affect the primordial abundances of light elements. Hence, baryogenesis, primordial nucleosynthesis and gravitino dark matter can be consistent in the range

$$
10^{-14}<\lambda, \lambda^{\prime}<10^{-7} .
$$


For a bino NLSP, a lifetime shorter than 0.1 s, i.e., couplings $\lambda, \lambda^{\prime}>10^{-12}$ are required by consistency with BBN.

The analysis of constraints on the superpotential terms (2.1) can be extended to general R-parity breaking mass terms [17], yielding again a range of allowed parameters. One finds that possible contributions to neutrino masses are negligable, once the cosmological constraints are satisfied.

Decaying gravitino dark matter can contribute to the EGRET and HEAT anomalies for a gravitino lifetime $\tau_{3 / 2} \sim 10^{26}$ s. For a gravitino mass $m_{3 / 2} \sim 10 \mathrm{GeV}$, and assuming $\eta \simeq 1 \mathrm{in} \mathrm{Eq.} \mathrm{(2.3),} \mathrm{this} \mathrm{requires} \mathrm{R-parity} \mathrm{violating} \mathrm{couplings} \lambda \sim 10^{-7}$. As we shall see, universal boundary conditions for gaugino masses favour larger gravitino masses, in the range

$$
10 \mathrm{GeV}<m_{3 / 2}<500 \mathrm{GeV},
$$

which, for fixed gravitino lifetime and $\eta \sim 1$, corresponds to the range of R-parity violating couplings

$$
10^{-10}<\lambda<10^{-7} \text {. }
$$

Note that for couplings below $\sim 10^{-9}$, most NLSPs decay outside the detector. However, for couplings above $\sim 10^{-11}$, corresponding to lifetimes shorter than $\sim 10^{-3} \mathrm{~s}$, some NLSP decays may still be observable in the detector [22].

How can the phenomenologically required small R-parity violating couplings arise? In [13] an example was presented, where the spontaneous breaking of R-parity is tied to B-L breaking. Recently, it has been shown that also the breaking of left-right symmetry can lead to small R-parity breaking [23].

\section{Thermal leptogenesis}

Let us now consider standard thermal leptogenesis as the source of the cosmological baryon asymmetry. In the high-temperature phase of the early universe thermally produced righthanded neutrinos generate an asymmetry in B-L, which leads to a baryon asymmetry via sphaleron processes. In the case of hierarchical right-handed neutrinos, and neglecting flavour effects, the baryon density relative to the photon density is given by (cf. [2])

$$
\frac{n_{B}}{n_{\gamma}} \simeq-1.04 \times 10^{-2} \epsilon_{1} \kappa,
$$

where $\epsilon_{1}$ is the CP asymmetry in the decay of the lightest right-handed neutrino $N_{1}$ into a pair of lepton $(L)$ and Higgs $\left(H_{u}\right)$ doublets, the efficiency factor $\kappa$ represents the effects of washout and scattering processes, and we have assumed a supersymmetric thermal plasma. The CP asymmetry $\epsilon_{1}$ satisfies an upper bound because of the seesaw relation, which for supersymmetric models reads [24-26],

$$
\left|\epsilon_{1}\right| \equiv\left|\frac{\Gamma\left(N_{1} \rightarrow L+H_{u}\right)-\Gamma\left(N_{1} \rightarrow L^{c}+H_{u}^{c}\right)}{\Gamma\left(N_{1} \rightarrow L+H_{u}\right)+\Gamma\left(N_{1} \rightarrow L^{c}+H_{u}^{c}\right)}\right| \lesssim \frac{3 M_{1}}{8 \pi\left\langle H_{u}\right\rangle^{2}} \frac{\Delta m_{\mathrm{atm}}^{2}}{m_{1}+m_{3}} .
$$


Here $m_{i}$, with $m_{1}<m_{2}<m_{3}$, are the mass eigenvalues of the light neutrinos and $M_{1}$ is the mass of the right-handed neutrino $N_{1}$. The atmospheric neutrino mass squared difference is determined from neutrino oscillation experiments as $\Delta m_{\mathrm{atm}}^{2} \simeq(2.5 \pm 0.2) \times 10^{-3} \mathrm{eV}^{2}$. Note that the upper bound on $\left|\epsilon_{1}\right|$, and therefore the maximally generated baryon asymmetry, increases proportional to the heavy Majorana mass $M_{1}$.

The efficiency factor $\kappa$ has to be determined by solving the Boltzmann equations. In the most interesting case of zero initial abundance of the right-handed neutrinos one finds for its maximal value, with and without supersymmetry, $\kappa \simeq 0.2$ [27,28]. Using (3.2), one then obtains from the observed baryon asymmetry [29],

$$
\frac{n_{B}}{n_{\gamma}}=(6.21 \pm 0.16) \times 10^{-10},
$$

the lower bound on the right-handed neutrino mass

$$
M_{1} \gtrsim 1.4 \times 10^{9} \mathrm{GeV}\left(\frac{\left\langle H_{u}\right\rangle}{174 \mathrm{GeV}}\right)^{2}
$$

at the $3 \sigma$ level of $n_{B} / n_{\gamma}$ and $\Delta m_{\mathrm{atm}}^{2}$. The corresponding lower bound on the reheating temperature is about a factor two smaller [30]. In the following analysis we shall therefore use as an estimate

$$
T_{R} \gtrsim 1 \times 10^{9} \mathrm{GeV} \text {. }
$$

Note that this bound on the reheating temperature only applies for hierarchical righthanded neutrinos. In the case of quasi-degenerate heavy neutrinos it is relaxed. The bound also assumes thermal equilibrium, and it is modified once the reheating process is taking into account. For instance, in the case of reheating by inflaton decays, the bound increases by about a factor of two [28].

Relic gravitinos with masses larger than $1 \mathrm{GeV}$ contribute to cold dark matter. In the following analysis we identify the thermally produced abundance $\Omega_{3 / 2} h^{2}$ with the $2 \sigma$ upper bound on the dark matter abundance deduced from the CMB anisotropies. From the WMAP 5-year results one obtains [29],

$$
\Omega_{3 / 2} h^{2} \equiv \Omega_{\mathrm{DM}} h^{2} \simeq 0.1223 .
$$

The thermal production of gravitinos is dominated by QCD processes. To leading order in the gauge coupling we find

$$
\Omega_{3 / 2} h^{2} \simeq 0.5\left(\frac{100 \mathrm{GeV}}{m_{3 / 2}}\right)\left(\frac{m_{\text {gluino }}}{1 \mathrm{TeV}}\right)^{2}\left(\frac{T_{R}}{10^{10} \mathrm{GeV}}\right)
$$

where $m_{\text {gluino }}$ is the physical gluino mass. Note that the coefficient 1 is about a factor two larger than in the analysis [31]. This is due to the 2-loop running of the gluino mass, which has been taken into account. Electroweak contributions to thermal gravitino production

\footnotetext{
${ }^{1}$ Varying superparticle masses (cf. Section 4 ), the value can change by about $10 \%$.
} 
further increase the abundance by about 20\%. In our numerical analysis we shall take this into account following [32]. Note that the gravitino production rate has an $\mathcal{O}(1)$ uncertainty due to unknown higher order contributions and nonperturbative effects [31]. Resummation of thermal masses increases the production rate by about a factor of two [33]. We also neglect nonthermal contributions to gravitino production, in particular from inflaton decay [34], which are usually subdominant at the considered high temperatures.

Our main interest are constraints on gluino and NLSP masses for gravitino dark matter. It is then convenient to rewrite (3.7) as

$$
m_{\mathrm{NLSP}} \simeq 310 \mathrm{GeV}\left(\frac{\xi}{0.2}\right)\left(\frac{m_{3 / 2}}{100 \mathrm{GeV}}\right)^{1 / 2}\left(\frac{10^{9} \mathrm{GeV}}{T_{R}}\right)^{1 / 2}, \quad \xi=\frac{m_{\mathrm{NLSP}}}{m_{\text {gluino }}},
$$

where the ratio $\xi$ is fixed by the boundary conditions of the soft supersymmetry breaking parameters. For each gravitino mass and reheating temperature, Eq. (3.8) then gives the NLSP mass for which the observed dark matter density is obtained. The maximal NLSP mass is reached for $m_{3 / 2}=m_{\mathrm{NLSP}}$,

$$
m_{\mathrm{NLSP}} \lesssim 980 \mathrm{GeV}\left(\frac{\xi}{0.2}\right)^{2}\left(\frac{10^{9} \mathrm{GeV}}{T_{R}}\right)
$$

In this paper, we focus on thermally produced gravitino dark matter. A high reheating temperature can also be consistent with leptogenesis in the case of very heavy gravitinos, as in anomaly mediation [35] or mirage mediation [36,37]. In those models, the gravitino can have a mass of about $100 \mathrm{TeV}$ and thus decays before BBN starts. However, these models have several intrinsic difficulties. In the case of anomaly mediation, it is difficult to explain the $g-2$ anomaly together with the $b \rightarrow s \gamma$ constraint, since the gaugino masses are controlled by the beta functions. In mirage mediation models, one often has a light modulus field whose decay produces too many gravitinos [38]. Hence, the heavy gravitino scenario appears to be phenomenologically disfavoured.

\section{Models and low-energy observables}

In order to illustrate the implications of leptogenesis and gravitino dark matter on superparticle masses, we now study two typical boundary conditions for the supersymmetry breaking parameters of the MSSM at the grand unification (GUT) scale:

$$
\text { (A) } m_{0}=m_{1 / 2}, \quad a_{0}=0, \quad \tan \beta
$$

with equal universal scalar and gaugino masses, $m_{0}$ and $m_{1 / 2}$, respectively; in this case a bino-like neutralino becomes the NLSP. The second boundary condition is

$$
\text { (B) } m_{0}=0, m_{1 / 2}, \quad a_{0}=0, \quad \tan \beta,
$$

which yields the right-handed stau as NLSP. In both cases, the trilinear scalar coupling $a_{0}$ is put to zero for simplicity. The ratio $\tan \beta$ of the Higgs vacuum expectation values and the 
universal gaugino mass $m_{1 / 2}$ are the two remaining independent variables. Superparticle masses at the electroweak scale are obtained by solving the renormalization group equations at 2-loop accuracy by means of SOFTSUSY 2.0.18 [39].

Low-energy observables yield a lower bound on superparticle masses. Since the thermal gravitino abundance (3.7) increases quadratically with the gluino mass, this implies an upper bound on the reheating temperature. Together with the lower bound from leptogenesis one then obtains a range of allowed reheating temperatures. In the same way, leptogenesis and gravitino dark matter yield an upper bound on superparticle masses. Combined with low-energy constraints, a window of allowed superparticle masses is obtained.

One of the strongest constraints on the MSSM parameter space follows from the lower bound on the Higgs boson mass by LEP [40],

$$
m_{h}>114.4 \mathrm{GeV} \text { (95\%C.L.) . }
$$

The bound is satisfied by enhancing radiative corrections to the Higgs potential, which requires a large stop mass. The parameters of the stop sector are essentially controlled by the gluino mass, i.e. $m_{1 / 2}$, via the renormalization group evolutions; they are less sensitive to the scalar mass $m_{0}$. The potential is also affected by the trilinear stop coupling $A_{t}$ for sufficiently large $a_{0}$. Although we put $a_{0}=0$ in the numerical analysis, we shall comment

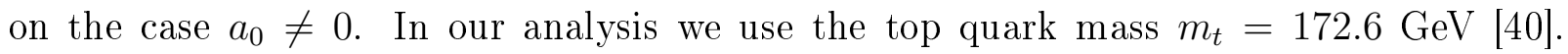
Radiative corrections are taken into account at the 2-loop level by means of FeynHiggs 2.6.4 [41].

When the superparticles are light, they contribute significantly to rare processes. The measured branching ratio $\operatorname{Br}\left(B_{d} \rightarrow X_{s} \gamma\right)$ agrees with the SM prediction. The SUSY contributions are dominated by the top-charged Higgs and stop-chargino diagrams. The latter is enhanced by large $\tan \beta$ and interferes with the former. In our analysis we choose the sign of the supersymmetric Higgs mass parameter $\mu_{H}$ such that the effect of the SUSY contributions is reduced. Taking into account the theoretical uncertainties, we require for the full MSSM prediction the conservative upper and lower bounds,

$$
2 \times 10^{-4}<\operatorname{Br}\left(B_{d} \rightarrow X_{s} \gamma\right)<4 \times 10^{-4} \text {. }
$$

The numerical analysis is based on SusyBSG 1.1.2 which takes NNLO contributions partially into account [42].

The two observables discussed above constrain the MSSM parameters. In contrast, the apparent discrepancy between the measured value of the muon anomalous magnetic moment [43] and the SM prediction may be an effect of supersymmetry, which then favours a certain range of MSSM parameters. Recently, the hadronic contribution to the SM prediction has been updated using $e^{+} e^{-}$data [44]. The current discrepancy with experiment is given by [45]

$$
a_{\mu}(\exp )-a_{\mu}(\mathrm{SM})=302(88) \times 10^{-11},
$$

which corresponds to a $3.4 \sigma$ deviation. An explanation of this discrepancy by hypothetical errors in the determination of the hadronic SM contribution appears unlikely [45]. In contrast, supersymmetry can easily account for the discrepancy [46]. The SUSY contribution 
is proportional to $\tan \beta$ and depends on $\operatorname{sgn}\left(\mu_{H}\right)$. It is remarkable that the deviation from the SM prediciton for $a_{\mu}$ and the agreement for $\operatorname{Br}\left(B_{d} \rightarrow X_{s} \gamma\right)$ require the same $\operatorname{sgn}\left(\mu_{H}\right)$ in the case of universal gaugino masses at the GUT scale. In the following, we use FeynHiggs to evaluate the SUSY contribution to $a_{\mu}$ at the 2-loop level.

Finally, the absense of pair production of heavy charged particles at LEP implies the approximate lower mass bound [40]

$$
m_{\text {charged }}>100 \mathrm{GeV} .
$$

In the next section we shall use superparticle masses obtained by means of SOFTSUSY.

\section{$5 \quad$ Numerical analysis}

We are now ready to determine the superparticle mass window and the allowed range of reheating temperatures for the two examples of universal boundary conditions at the GUT scale, which were discussed in the previous section.

In Fig. 1 the upper bound (3.9) on the NLSP masses is shown for reheating temperatures $T_{R} \geq 1 \times 10^{9} \mathrm{GeV}$, which is the lower bound required by leptogenesis. In case (A) with bino NLSP, the ratio $\xi=m_{\mathrm{NLSP}} / m_{\text {gluino }}$, and therefore the upper bound on $m_{\mathrm{NLSP}}$, are essentially independent of $\tan \beta$. In contrast, for (B) with stau NLSP, one has a strong dependence on $\tan \beta$. The lower bound on $m_{\mathrm{NLSP}}$ is determined by $\operatorname{Br}\left(B_{d} \rightarrow X_{s} \gamma\right)$ and the Higgs mass bound in case (A), and the charged particle and Higgs mass bounds in case (B), respectively. We find the allowed mass ranges

$$
\text { (A) } 130 \mathrm{GeV}<m_{\text {bino }}<620 \mathrm{GeV}, \quad \text { (B) } 100 \mathrm{GeV}<m_{\text {stau }}<490 \mathrm{GeV} \text {. }
$$

Note that in case (B) upper and lower bounds correspond to different values of $\tan \beta$. The muon g-2 anomaly favours small NLSP masses in the range from $100 \mathrm{GeV}$ to $300 \mathrm{GeV}$. One also obtains upper bounds on the gravitino mass,

$$
\text { (A) } m_{3 / 2}<620 \mathrm{GeV}, \quad \text { (B) } m_{3 / 2}<490 \mathrm{GeV} \text {. }
$$

Both boundary conditions have $a_{0}=0$. For negative $a_{0}$, the Higgs boson potential is modified in such a way that the dashed line in Fig. 1 moves to the left. We have checked that the reheating temperature can then reach $6 \times 10^{9} \mathrm{GeV}$, whereas other observables are not much affected. We therefore obtain for the range of reheating temperatures consistent with leptogenesis and gravitino dark matter

$$
T_{R}=(1-6) \times 10^{9} \mathrm{GeV} .
$$

Note that according to FeynHiggs, the theoretical uncertainty of the Higgs boson is about $1 \mathrm{GeV}$ for $m_{h} \simeq 115 \mathrm{GeV}$. This corresponds to an uncertainty of $10-20 \%$ for the upper bound on the reheating temperature. 


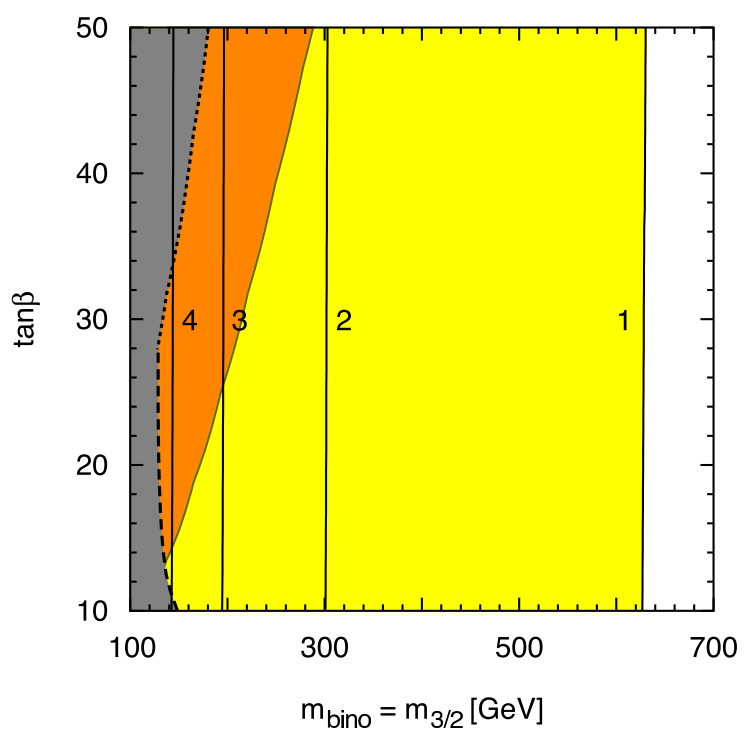

(a)

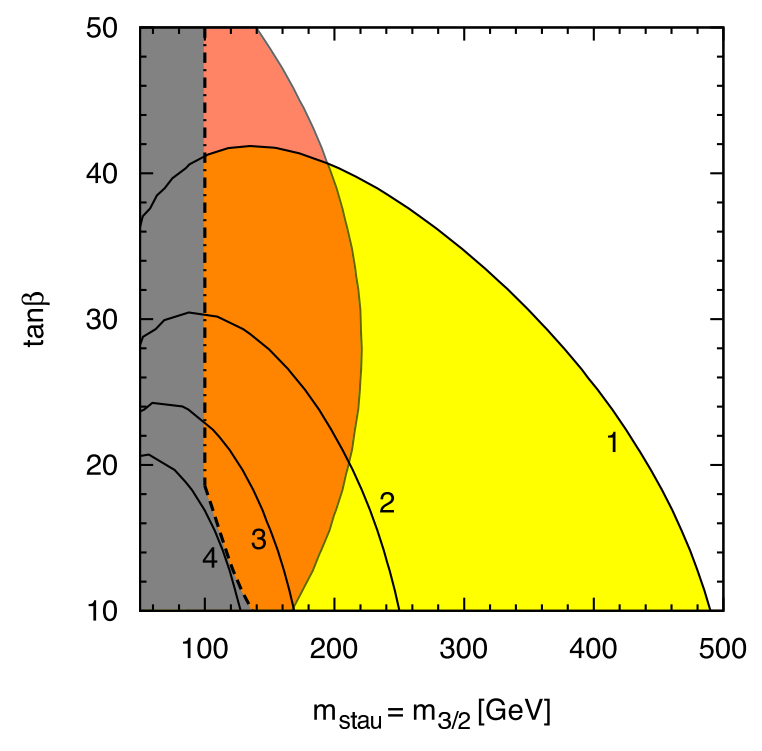

(b)

Figure 1: Contours of constant reheating temperature, $T_{R}=(1-4) \times 10^{9} \mathrm{GeV}$, with $\Omega_{3 / 2}=\Omega_{\mathrm{DM}}$ (solid lines) (cf. Eq. (3.6)). The panels (a) and (b) correspond to the GUT boundary conditions (A) and (B) with bino-like NLSP and stau NLSP, respectively. The choice $m_{3 / 2}=m_{\mathrm{NLSP}}$ maximizes the reheating temperature. The gray region is excluded by constraints from low-energy experiments: the lower $\tan \beta$ part (left of the dashed line) does not satisfy the LEP Higgs mass bound; the higher $\tan \beta$ part in (a) (left of the dotted line) is ruled out by $\operatorname{Br}\left(B_{d} \rightarrow X_{s} \gamma\right)$; the higher $\tan \beta$ part in (b) (left of the dot-dashed line) does not satisfy the lower mass bound on charged particles from LEP. Thermal leptogenesis is possible in the yellow and orange regions; the orange region is favored by the muon $g-2$ anomaly at the $2 \sigma$ level.

We can also study superparticle masses as function of gravitino mass and reheating temperature using Eq. (3.8). The allowed NLSP mass range then depends on $\tan \beta$. In the case of bino NLSP, consider as an example

$$
\text { (A) } \tan \beta=30, \quad \xi=\frac{m_{\text {bino }}}{m_{\text {gluino }}}=0.17-0.19 .
$$

The left panel of Fig. 2 shows the bino mass yielding the observed dark matter abundance as function of the gravitino mass for different reheating temperatures; the right panel is the corresponding plot for the gluino mass. Upper mass bounds are obtained for the smallest temperature of $1 \times 10^{9} \mathrm{GeV}$ and the largest gravitino mass $m_{3 / 2}=m_{\text {bino, }}$,

$$
\text { (A) } \tan \beta=30: m_{\text {bino }} \lesssim 620 \mathrm{GeV}, \quad m_{\text {gluino }} \lesssim 3.1 \mathrm{TeV} .
$$

For smaller gravitino masses the bounds become more stringent. For instance, for $m_{3 / 2}=$ $100 \mathrm{GeV}$, one obtains

$$
m_{\text {bino }} \lesssim 270 \mathrm{GeV}, \quad m_{\text {gluino }} \lesssim 1.5 \mathrm{TeV} .
$$




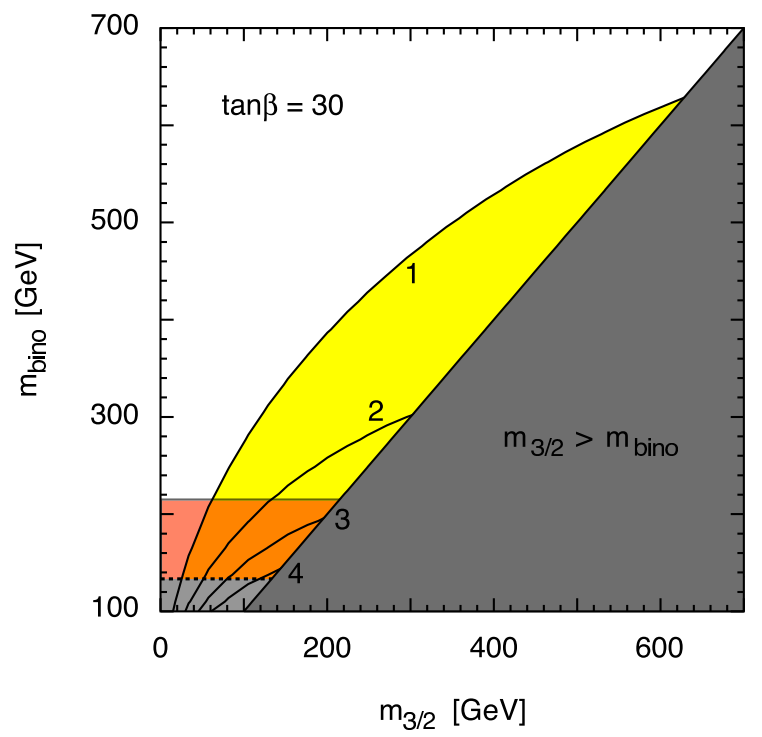

(a)

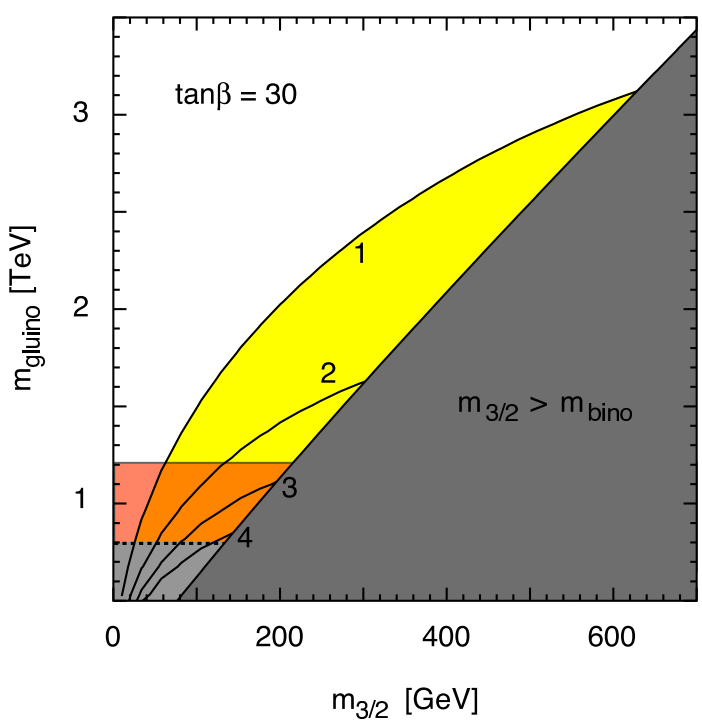

(b)

Figure 2: Contours of constant reheating temperature in the $m_{\text {bino }}-m_{3 / 2}$ plane (a) and the $m_{\text {gluino }}-m_{3 / 2}$ plane (b) for boundary condition (A) with bino NLSP (see caption of Fig. 1 for details). In the dark gray region, the gravitino is not the LSP.

Note that these bounds are essentially independent of $m_{0}$ and $\tan \beta$, als long as $m_{0} \sim m_{3 / 2}$.

In the case of stau NLSP, there is a strong dependence on $\tan \beta$. As an example, we consider

$$
\text { (B) } \tan \beta=10, \quad \xi=\frac{m_{\text {stau }}}{m_{\text {gluino }}}=0.16-0.17 .
$$

$\xi$ decreases with increasing $\tan \beta$. Stau and gluino masses are shown in Fig. 3. Since the ratio of NLSP and gluino mass is smaller, the mass bounds are now more stringent,

$$
\text { (B) } \tan \beta=10: m_{\text {stau }} \lesssim 490 \mathrm{GeV}, \quad m_{\text {gluino }} \lesssim 2.8 \mathrm{TeV} \text {. }
$$

For a gravitino mass $m_{3 / 2}=100 \mathrm{GeV}$, one obtains

$$
m_{\text {stau }} \lesssim 240 \mathrm{GeV}, \quad m_{\text {gluino }} \lesssim 1.5 \mathrm{TeV} .
$$

Let us emphasize again the effect of the theoretical uncertainty in the evaluation of the gravitino abundance, which is expected to be $\mathcal{O}(1)$ [31]. For instance, if the gravitino production rate is larger by a factor 2, as suggested in [33], all reheating temperatures in Figs. 1, 22 and 3 are by a factor 2 smaller. Hence, the superparticle mass range consistent with thermal leptogenesis becomes narrower. On the other hand, a smaller gravitino production rate would enlarge the parameter range consistent with leptogenesis.

Finally, let us comment on other boundary conditions. We have chosen universal gaugino masses, with $m_{0}=m_{1 / 2}$ or $m_{0}=0$ at the GUT scale. However, even for non-universal gaugino masses we obtain almost the same results. The reason is that all the bounds are 


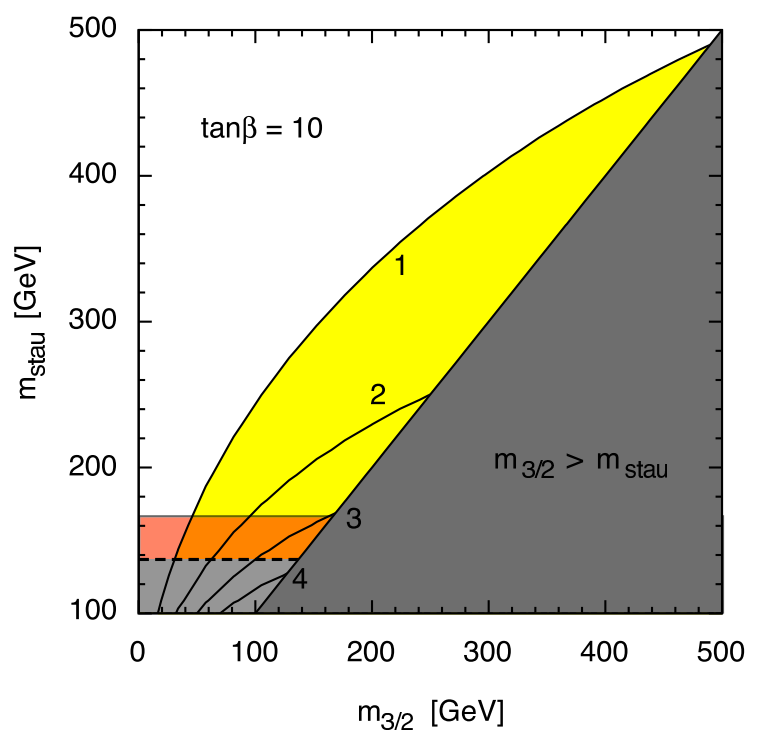

(a)

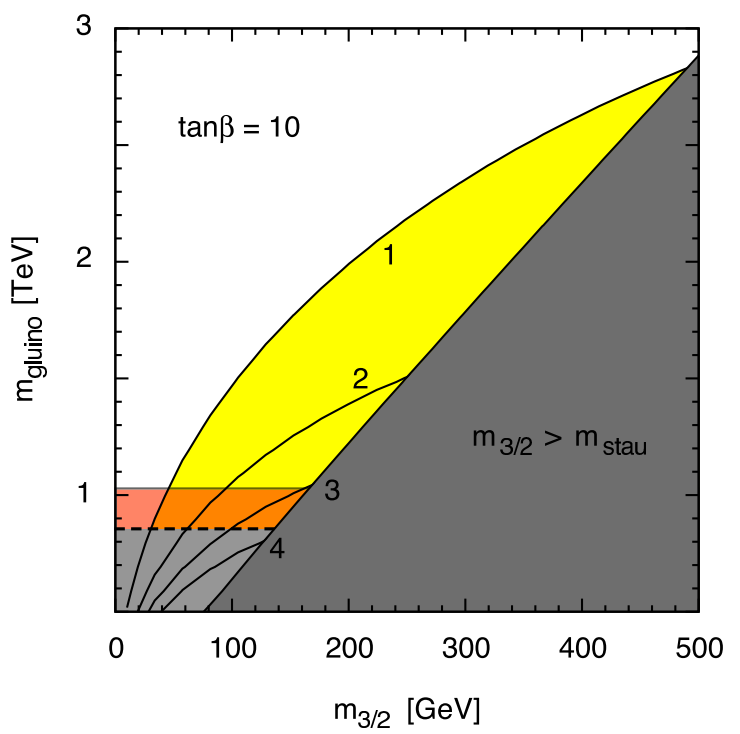

(b)

Figure 3: Contours of constant reheating temperature in the $m_{\text {stau }}-m_{3 / 2}$ plane (a) and the $m_{\text {gluino }}-m_{3 / 2}$ plane (b) for boundary condition (B) with stau NLSP (see caption of Fig. 1 for details). In the dark gray region, the gravitino is not the LSP.

controlled by the gluino mass. Reducing the gluino mass, the dark matter bound on the reheating temperature is relaxed, but the low-energy constraints become severer: supersymmetric contributions to the Higgs boson mass are suppressed, while they are enhanced for $\operatorname{Br}\left(B_{d} \rightarrow X_{s} \gamma\right)$. As a consequence, the maximal reheating temperature remains almost the same as in the case of universal gaugino masses. On the other hand, the low-energy constraints become weaker for scalar masses much larger than $m_{1 / 2}$. One can then reach reheating temperatures $\sim 10^{10} \mathrm{GeV}$.

\section{Conclusions and outlook}

We have studied the implications of thermal leptogenesis and gravitino dark matter for the mass spectrum of superparticles. In the case of broken R-parity the constraints from nucleosynthesis are naturally fulfilled, and universal gaugino masses at the GUT scale are possible, contrary to the case of stable gravitinos.

As an illustration, we have considered two boundary conditions which lead to a bino-like NLSP and a stau NLSP, respectively. Low-energy observables and gravitino dark matter together with thermal leptogenesis yield upper and lower bounds on NLSP and gluino masses, which in both cases lie within the discovery range of the LHC. It is encouraging that the supersymmetric explanation of the muon $g-2$ anomaly favours smaller masses within these mass windows.

A cosmology with leptogenesis and gravitino dark matter also leads to the prediction 
of a maximal temperature in the early universe. In the case of universal gaugino masses at the unification scale we find the upper bound $T_{R}^{\max } \simeq 6 \times 10^{9} \mathrm{GeV}$, which is somewhat relaxed for large scalar masses. This bound has been obtained under the assumption of thermal equilibrium, which appears unlikely for a maximal temperature. Nevertheless, it is intriguing that the temperature $T_{R}^{\max }$ is of the same order of magnitude as the critical for the destabilization of compact dimensions in higher-dimensional supersymmetric theories [47]. The effect of the reheating process on the stabilization of extra dimensions and the relation to baryogenesis and dark matter require futher investigations.

Gravitino decays produce a flux of photons and positrons, which can significantly contribute to the EGRET and HEAT anomalies for a lifetime $\tau_{3 / 2} \sim 10^{26} \mathrm{~s}$. If these anomalies are indeed related to gravitino decays, the satellite experiments FGST and PAMELA should soon detect characteristic features in the photon and positron spectrum, respectively. Observation of a line in the gamma-ray spectrum by FGST and a rise with sharp cutoff in the positron spectrum by PAMELA would lead to a determination of the gravitino mass. This would considerably tighten the predictions for superparticle mass windows which will be probed at the LHC.

\section{References}

[1] M. Fukugita and T. Yanagida, Phys. Lett. B 174, 45 (1986).

[2] For a review and references, see W. Buchmuller, R. D. Peccei and T. Yanagida, Ann. Rev. Nucl. Part. Sci. 55 (2005) 311; S. Davidson, E. Nardi and Y. Nir, arXiv:0802.2962 [hep-ph].

[3] S. Weinberg, Phys. Rev. Lett. 48 (1982) 1303.

[4] J. R. Ellis, D. V. Nanopoulos and S. Sarkar, Nucl. Phys. B 259 (1985) 175.

[5] M. Kawasaki, K. Kohri and T. Moroi, Phys. Lett. B 625, 7 (2005); Phys. Rev. D 71, 083502 (2005); K. Jedamzik, Phys. Rev. D 74, 103509 (2006).

[6] T. Moroi, H. Murayama and M. Yamaguchi, Phys. Lett. B 303, 289 (1993).

[7] M. Bolz, W. Buchmuller and M. Plumacher, Phys. Lett. B 443 (1998) 209.

[8] M. Pospelov, Phys. Rev. Lett. 98, 231301 (2007).

[9] M. Ratz, K. Schmidt-Hoberg and M. W. Winkler, arXiv:0808.0829 [hep-ph]; J. Pradler and F. D. Steffen, arXiv:0808.2462 [hep-ph].

[10] T. Kanzaki, M. Kawasaki, K. Kohri and T. Moroi, Phys. Rev. D 75, 025011 (2007).

[11] J. L. Diaz-Cruz, J. R. Ellis, K. A. Olive and Y. Santoso, JHEP 0705 (2007) 003; C. F. Berger, L. Covi, S. Kraml and F. Palorini, JCAP 0810 (2008) 005. 
[12] B. A. Campbell, S. Davidson, J. R. Ellis and K. A. Olive, Phys. Lett. B 256, 484 (1991); W. Fischler, G. F. Giudice, R. G. Leigh and S. Paban, Phys. Lett. B 258, 45 (1991); H. K. Dreiner and G. G. Ross, Nucl. Phys. B 410, 188 (1993).

[13] W. Buchmuller, L. Covi, K. Hamaguchi, A. Ibarra and T. Yanagida, JHEP 0703, 037 (2007).

[14] F. Takayama and M. Yamaguchi, Phys. Lett. B 485, 388 (2000).

[15] G. Bertone, W. Buchmuller, L. Covi and A. Ibarra, JCAP 0711 (2007) 003.

[16] A. Ibarra and D. Tran, Phys. Rev. Lett. 100, 061301 (2008).

[17] K. Ishiwata, S. Matsumoto and T. Moroi, arXiv:0805.1133 [hep-ph].

[18] A. Ibarra and D. Tran, JCAP 0807 (2008) 002.

[19] A. W. Strong, I. V. Moskalenko and O. Reimer, Astrophys. J. 613, 956 (2004); Astrophys. J. 613 (2004) 962.

[20] S. W. Barwick et al. [HEAT Collaboration], Astrophys. J. 482, L191 (1997).

[21] B. C. Allanach, A. Dedes and H. K. Dreiner, Phys. Rev. D 69 (2004) 115002 [Erratumibid. D 72 (2005) 079902]; R. Barbier et al., Phys. Rept. 420, 1 (2005).

[22] K. Ishiwata, T. Ito and T. Moroi, arXiv:0807.0975 [hep-ph].

[23] X. Ji, R. N. Mohapatra, S. Nussinov and Y. Zhang, arXiv:0808.1904 [hep-ph].

[24] S. Davidson and A. Ibarra, Phys. Lett. B 535 (2002) 25.

[25] K. Hamaguchi, H. Murayama and T. Yanagida, Phys. Rev. D 65, 043512 (2002).

[26] L. Covi, E. Roulet and F. Vissani, Phys. Lett. B 384, 169 (1996).

[27] W. Buchmuller, P. Di Bari and M. Plumacher, Annals Phys. 315, 305 (2005).

[28] G. F. Giudice, A. Notari, M. Raidal, A. Riotto and A. Strumia, Nucl. Phys. B 685, 89 (2004).

[29] G. Hinshaw et al. [WMAP Collaboration], arXiv:0803.0732 [astro-ph].

[30] S. Blanchet and P. Di Bari, JCAP 0703 (2007) 018 arXiv:hep-ph/0607330.

[31] M. Bolz, A. Brandenburg and W. Buchmuller, Nucl. Phys. B 606, 518 (2001) [Erratum-ibid. B 790, 336 (2008)].

[32] J. Pradler and F. D. Steffen, Phys. Rev. D 75, 023509 (2007).

[33] V. S. Rychkov and A. Strumia, Phys. Rev. D 75, 075011 (2007). 
[34] M. Endo, F. Takahashi and T. T. Yanagida, Phys. Rev. D 76 (2007) 083509.

[35] L. Randall and R. Sundrum, Nucl. Phys. B 557, 79 (1999); G. F. Giudice, M. A. Luty, H. Murayama and R. Rattazzi, JHEP 9812, 027 (1998).

[36] K. Choi, A. Falkowski, H.P. Nilles and M. Olechowski, Nucl. Phys. B718 (2005) 113.

[37] M. Endo, M. Yamaguchi and K. Yoshioka, Phys. Rev. D72 (2005) 015004; K. Choi, K.S. Jeong and K.i. Okumura, JHEP 0509 (2005) 039.

[38] M. Endo, K. Hamaguchi and F. Takahashi, Phys. Rev. Lett. 96 (2006) 211301; S. Nakamura and M. Yamaguchi, Phys. Lett. B 638 (2006) 389.

[39] B. C. Allanach, Comput. Phys. Commun. 143, 305 (2002).

[40] C. Amsler et al. (Particle Data Group), Physics Letters B667, 1 (2008)

[41] M. Frank, T. Hahn, S. Heinemeyer, W. Hollik, H. Rzehak and G. Weiglein, JHEP 0702, 047 (2007); G. Degrassi, S. Heinemeyer, W. Hollik, P. Slavich and G. Weiglein, Eur. Phys. J. C 28, 133 (2003); S. Heinemeyer, W. Hollik and G. Weiglein, Eur. Phys. J. C 9, 343 (1999); Comput. Phys. Commun. 124, 76 (2000).

[42] G. Degrassi, P. Gambino and P. Slavich, arXiv:0712.3265 [hep-ph].

[43] G. W. Bennett et al. [Muon G-2 Collaboration], Phys. Rev. D 73, 072003 (2006).

[44] K. Hagiwara, A. D. Martin, D. Nomura and T. Teubner, Phys. Lett. B 649, 173 (2007).

[45] For a recent discussion and references, see M. Passera, W. J. Marciano and A. Sirlin, arXiv:0804.1142 [hep-ph]; arXiv:0809.4062 [hep-ph].

[46] J.L. Lopez, D.V. Nanopoulos and X. Wang, Phys. Rev. D 49, 366 (1994); U. Chattopadhyay and P. Nath, Phys. Rev. D 53, 1648 (1996); T. Moroi, Phys. Rev. D 53, 6565 (1996); Erratum-ibid, D 56, 4424 (1997).

[47] W. Buchmuller, K. Hamaguchi, O. Lebedev and M. Ratz, Nucl. Phys. B 699 (2004) 292. 